\title{
Topological Indices for Nanoclusters
}

\author{
Forrest H. Kaatz, Mesalands Community College, Tucumcari, NM, USA \\ Adhemar Bultheel, Dept. Computer Sci., KU Leuven, Heverlee, Belgium
}

\begin{abstract}
Nanoclusters create the possibility of designing novel properties and devices based on finite structures, with small dimensions. Clusters are of interest for catalytic, optical, biochemical, and structural characteristics. We examine clusters of icosahedral, cuboctahedral, and decahedral symmetry. Examples of these types of structures are shown from gold and platinum nanoclusters. Starting with only the atomic coordinates, we create an adjacency and distance matrix that facilitates the calculation of topological indices, including the Wiener, hyper-Wiener, reverse Wiener, Szeged, Balaban, and Kirchhoff indices. Some of these indices correlate to properties of the cluster. We find these indices exhibit polynomial and inverse behavior as a function of an increasing number of shells. Additionally, all the indices can be modeled with power law curves, as a function of $N$, the number of atoms. The magnitude of the exponent in the power law is associated with an index. The asymptotic limits of the topological indices are determined as a function of $N$. A conjecture previously published on the asymptotic behavior of the Wiener index is experimentally confirmed.
\end{abstract}

\section{Introduction}

The phrase 'nanocluster' is often used to describe nanosized particles, since they can form structures, which strictly speaking are not crystalline in that they do not have bulk symmetry, whereas a cluster sized form with dimensions of 1 to $100 \mathrm{~nm}$, may exist in the nano regime. There are many forms of shape and symmetry which may exist in nanosized materials [1], among them icosahedral, cuboctahedral, and decahedral.

Nanoclusters are created from atoms completing layers or shells sequentially giving rise to what has been phrased as 'magic numbers', or the number of atoms to complete a shell over a beginning layer. Thus, for some clusters, there has developed a large description of formulas, giving the relation of number of atoms, surface atoms, and bonds as a function of L, the number of shells in the cluster [2]. A summary of these formulas for icosahedral, cuboctahedral and decahedral clusters, for our purposes is given in Table 1. Note that the cuboctahedral clusters can be described as an FCC structure, whereas the icosahedral and decahedral cannot. We examine the topological indices of nanoclusters with these symmetries for sizes up to several thousand atoms. 


\begin{tabular}{|c|c|c|c|c|}
\hline Definition & Notation & ICO & CO $($ FCC $)$ & DECA \\
\hline \# atoms & $N$ & $10 \frac{L^{3}}{3}+5 L^{2}+11 \frac{L}{3}+1$ & $\begin{array}{l}10 \frac{L^{3}}{3}+5 L^{2}+11 \frac{L}{3}+1(L \text { even }) \\
10 \frac{L^{3}}{3}+3 L^{2}-\frac{L}{3}(L \text { odd })\end{array}$ & $\frac{L}{6}$ \\
\hline \# surface at. & $N_{S}$ & $10 L^{2}+2$ & $\begin{array}{l}10 L^{2}+2(L \text { even }) \\
10 L^{2}-4 L(L \text { odd })\end{array}$ & $5 L^{2}-10 L+7$ \\
\hline \# bonds & $N_{B}$ & $20 L^{3}+12 L^{2}+4 L$ & $\begin{array}{l}20 L^{3}+15 L^{2}+7 L(L \text { even }) \\
20 L^{3}-8 L(L \text { odd })\end{array}$ & $5 L^{3}-15 \frac{L^{2}}{2}+7 \frac{L}{2}-1$ \\
\hline
\end{tabular}

Table 1: Magic number formulas for the icosahedral, cuboctahedral, and decahedral structures. The first one has been published in references [3,4], while the cuboctahedral and decahedral ones are new.

A large collection of results from metal nanoclusters exists, as metals comprise 75elements in the Periodic Table. These results are fairly recent with the majority occurring since the beginning of this century 15 years ago from developments in chemical synthesis [3,4]. In Figure 1, we show reprints of gold and platinum nanoclusters having the icosahedral [5], cuboctahedral [6], and decahedral symmetry [7]. Gold and platinum clusters are especially of interest for catalytic purposes, as nano gold in particular has properties unknown in the bulk.

Topological indices can be traced back to the Wiener index in 1947 [8], although it was some time later in 1971 [9] that its status in the field of mathematical chemistry became established. Today there are many topological indices, and there have been fluctuations in the number of publications on these topics, with some indices becoming more relevant in the lexicon of topological chemistry. Recently, a $\mathrm{C}++$ program detailing the numerical calculation of many of these indices has been made available [10] and we have rewritten these programs with a MATLAB program that calculates the adjacency and distance matrices from only the atomic coordinates. It is worthwhile to note that the $\mathrm{C}++$ program only works for fullerenes, while our program only requires a coordinate file, and in principle any structure may be calculated. To be specific, any organic or inorganic structure where a coordinate file exists and where the nearest neighbors are well defined (do not overlap with second neighbors) may be calculated. The adjacency matrix is calculated from a subset of the list of neighbors, since that is how we may define it. In a different type of analysis, we have modeled both fullerenes and nanoclusters [11]. In 2D, we have also looked at Penrose tilings [12].

The only previous results on clusters we are aware of, are some calculations on nanoclusters of ZnS [13] which calculated the Wiener, hyper-Wiener and Szeged index. They also gave some correlations to properties of $\mathrm{ZnS}$ based on the topological indices. Although the property and design characteristics of molecular topology have been studied [14], our objective is to examine the computational modeling aspects of topological indices and nanoclusters. 

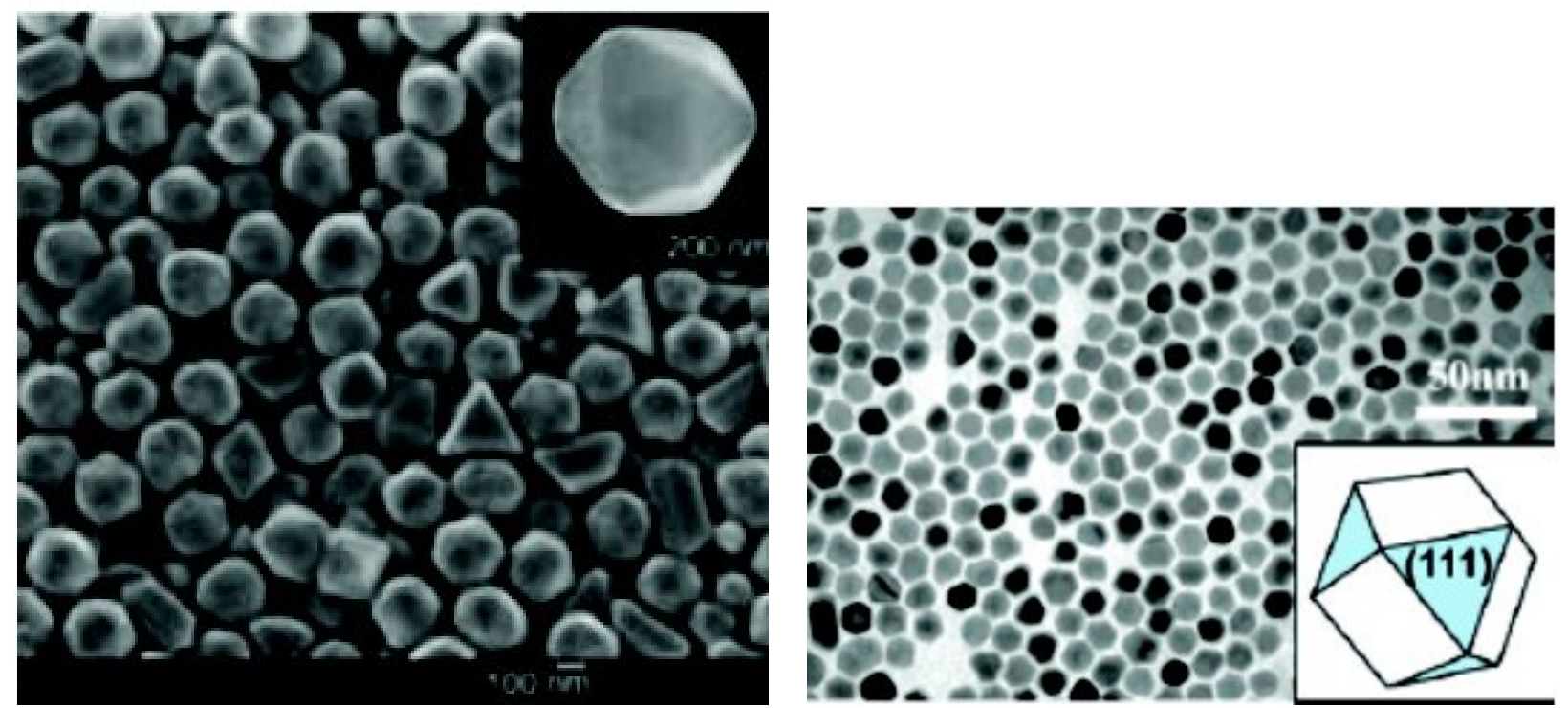

A. Gold Icosahedron [5]

B. Platinum Cuboctahedron [6]

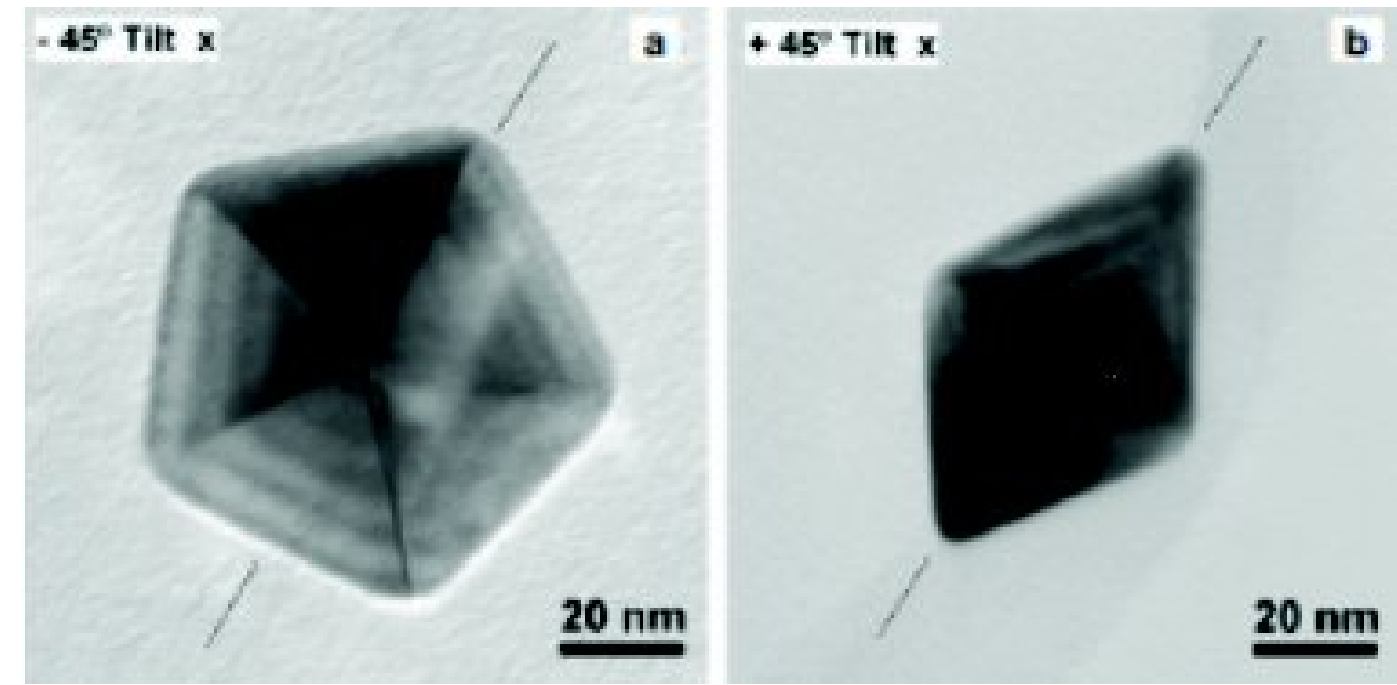

C. Gold Decahedron [7]

Figure 1: Nanostructures examined in this manuscript 


\section{Methods}

As mentioned, we use a theoretical graph-network approach, with atoms at the vertices, and links as nearest neighbor bonds. An adjacency matrix is created which contains a 1 at position $(i, j)$ if atom $j$ is in the set of nearest neighbors of atoms $i$, i.e., when the distance $r_{i j}$ is approximately equal to $r_{\min }=\min _{i \neq j} r_{i j}$. To allow for small deviations from the average bond length, we shall define $i$ and $j$ as nearest neighbors, and separate them from the rest by requiring that $r_{i j}<r_{c}$ where $r_{c}$ is a threshold value, appropriate for the nanocluster. Thus,

$$
\mathbf{A}(i, j)= \begin{cases}1 & \text { if } r_{i j}<r_{c} \text { and } i \neq j \\ 0 & \text { otherwise }\end{cases}
$$

As an alternative, we may consider the actual Euclidean distances in the adjacency matrix, i.e., replace every nonzero entry $\mathbf{A}(i, j)$ in the previous definition by the actual distance $r_{i j}$ and keep the zeros.

Some topological indices are most easily calculated from the distance matrix, which is defined as

$$
D_{i j}= \begin{cases}0, & i=j \\ d_{i j}, & i \neq j\end{cases}
$$

where $d_{i j}$ is the length of the shortest path from $i$ to $k$. An efficient algorithm for the calculation of the distance matrix from the adjacency matrix exists [15]. Using this definition, we can calculate the Wiener index, $W(G)$ and hyper-Wiener index, $W W(G)$ as

$$
\begin{gathered}
W(G)=\frac{1}{2} \sum_{i=1}^{n} \sum_{j=1}^{n} d_{i j}=\sum_{i>j=1}^{n} d_{i j} \\
W W(G)=\frac{1}{2} \sum_{i=1}^{n} \sum_{j=1}^{n}\left(d_{i j}+d_{i j}^{2}\right)
\end{gathered}
$$

and the reverse Wiener index $[16] \Lambda(G)$ is

$$
\Lambda(G)=r W=n(n-1) D / 2-W(G)
$$

where $D$ is the topological diameter defined as

$$
D=\max _{i}\left\{\max _{j}\left(D_{i j}\right)\right\}
$$

and $D_{i j}$ is an element of the distance matrix. A related index is the Szeged index introduced by Gutman [17], where

$$
S z(G)=\sum_{\text {edges }} n_{i} n_{j}
$$

where, for any edge $i j, n_{i}$ counts the vertices of $G$ that are closer to vertex $i$ than to $j$, and $n_{j}$ counts those that are closer to $j$ than to $i$, with vertices equidistant from $i$ and $j$ ignored. This is a natural generalization of the Wiener index since (6) is actually the formula used by 
Wiener for acyclic graphs (for which $W=S z$ ) [18]. The Szeged index also has some other similarities to the Wiener index, in that if $G$ and $G^{\prime}$ are catacondensed benzenoid systems with an equal number of hexagons, then $W(G)=W\left(G^{\prime}\right)(\bmod 8)$ and $S z(G)=S z\left(G^{\prime}\right)(\bmod 8)$ [19]. Also for any connected network $G, S z(G) \geq W(G)[20]$.

Another invariant derived from the distance matrix is the Balaban index [21-23], $J$,

$$
J(G)=\frac{b}{c+1} \sum_{\text {edges }}\left(d_{i} d_{j}\right)^{-1 / 2}
$$

where $b$ is the number of edges, $c$ is the number of primitive cycles and $d_{i}$ is the row sum

$$
d_{i}=\sum_{j=1}^{n} d_{i j}
$$

The Balaban index was originally introduced as being less degenerate than other indices [21], and has some asymptotic limits: for a linear alkane $C_{n} H_{2 n+2}$ it tends to $\pi$, while for highly branched alkanes, $J$ increases without limit.

The summed atomic displacement may be calculated from Kirchhoff index, and is valid for any graph $G$ [24]. The Kirchhoff index can be determined from the Laplacian matrix, where $\mathbf{L}$ is defined as

$$
\mathbf{L}=\Delta-\mathbf{A}
$$

and $\Delta$ is a diagonal matrix of order $n$, with diagonal elements $=$ the number of first neighbors, or the number of non-zero entries in a column in the adjacency matrix, $\mathbf{A}$ [25]. Then the Kirchhoff index is

$$
K f(G)=n \sum_{i=1}^{n-1} \frac{1}{\lambda_{i}}
$$

and $\lambda_{i}$ is an eigenvalue of $\mathbf{L}$. The summed atomic displacement is given as

$$
\sum_{i=1}^{n}\left(\Delta x_{i}\right)^{2}=\frac{1}{\beta k n} K f(G)
$$

where $k$ is the material nearest neighbor force constant, and $\beta$ is the inverse temperature [23].

\section{Results}

The MATLAB program generates 3D figures of the nanoclusters as shown in Figure 2. These figures are generated with the input data of the coordinates, and the calculated adjacency matrix from the MATLAB code. Figure $2 \mathrm{~A}$ shows an icosahedron with 55 atoms, $2 \mathrm{~B}$ shows a cuboctahedron with 55 atoms, and $2 \mathrm{C}$ shows a decahedron with 54 atoms. The calculations are quite rapid, taking a few seconds to less than 25 minutes for even the largest files of around 7,000 atoms on a computer with an i7 processor, 32GB of RAM, and running 

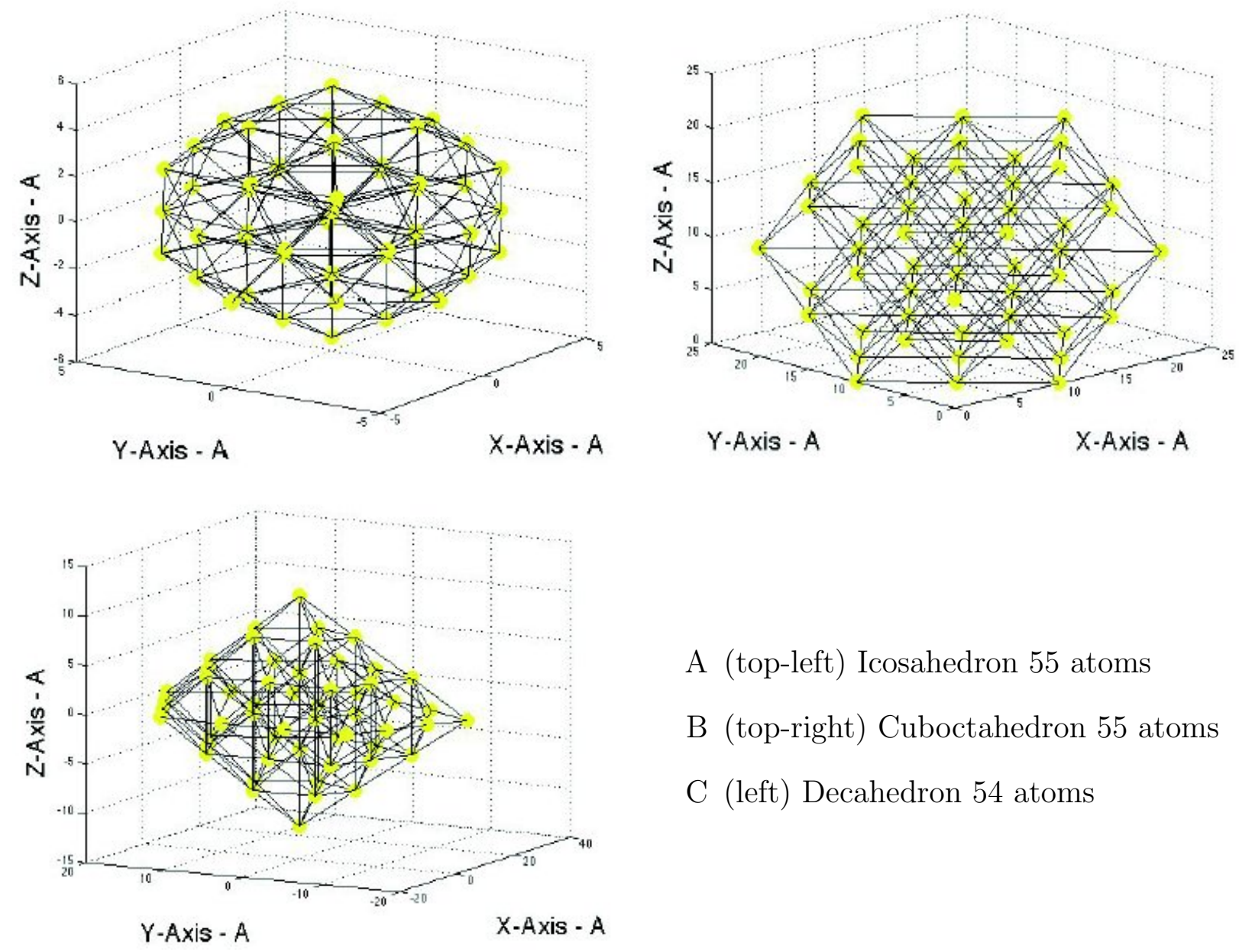
A (top-left) Icosahedron 55 atoms
B (top-right) Cuboctahedron 55 atoms
C (left) Decahedron 54 atoms

Figure 2: Nanostructures examined in this manuscript. 


\begin{tabular}{|c|c|c|c|c|c|c|c|}
\hline$C_{60}$ & $N$ & $W$ & $W W$ & $r W$ & $S z$ & $J$ & $K f$ \\
\hline & 60 & 8340 & 27180 & 7590 & 51840 & 0.9105215 & 2294.17225 \\
\hline CO-L & $N$ & $W$ & $W W$ & $r W$ & $S z$ & $J$ & $K f$ \\
\hline 1 & 6 & 18 & 21 & 12 & 48 & 3 & 6.5 \\
\hline 2 & 55 & 3840 & 7566 & 2100 & 31776 & 2.15695 & 519.13203 \\
\hline 3 & 116 & 22260 & 53646 & 17760 & 350130 & 1.80095 & 2114.50688 \\
\hline 4 & 309 & 223272 & 708912 & 157416 & 6464712 & 1.350241 & 14627.80004 \\
\hline 5 & 490 & 654942 & 2371671 & 543108 & 27196224 & 1.189396 & 35109.2898 \\
\hline 6 & 923 & 2891928 & 12644226 & 2214108 & 179667600 & 0.97653 & 122451.7282 \\
\hline 7 & 1288 & 6283848 & 30307356 & 5319744 & 501664608 & 0.886818 & 231774.7778 \\
\hline 8 & 2057 & 18817776 & 104770080 & 15015760 & 2028603048 & 0.763817 & 584033.525 \\
\hline 9 & 2670 & 34526826 & 207901449 & 29609244 & 4510898736 & 0.706875 & 965284.7774 \\
\hline 10 & 3871 & 82395984 & 557184870 & 67411716 & 13676169144 & 0.626912 & 2010793.293 \\
\hline 11 & 4796 & 135626172 & 978973578 & 117338848 & 26307168624 & 0.587263 & 3043952.646 \\
\hline 12 & 6525 & 268799700 & 2070597360 & 242029500 & 57113643600 & 0.554959 & 5411348.629 \\
\hline 13 & 7826 & 425559030 & 3580646667 & 370540820 & 114811934016 & 0.502805 & 7964038.84 \\
\hline ICO-L & $N$ & $W$ & $W W$ & $r W$ & $S z$ & $J$ & $K f$ \\
\hline 1 & 13 & 114 & 150 & 42 & 354 & 3.36428 & 25.382488 \\
\hline 2 & 55 & 3690 & 7080 & 2250 & 32970 & 2.340769 & 454.867103 \\
\hline 3 & 147 & 37596 & 94116 & 26790 & 646836 & 1.780042 & 3145.4653 \\
\hline 4 & 309 & 215100 & 663300 & 165588 & 6045810 & 1.432381 & 13503.46235 \\
\hline 5 & 561 & 869550 & 3184650 & 701250 & 36251160 & 1.19715 & 43482.71662 \\
\hline 6 & 923 & 2787174 & 11818680 & 2318862 & 161569044 & 1.027815 & 115500.0738 \\
\hline 7 & 1415 & 7567320 & 36458820 & 6438350 & 582313410 & 0.900236 & 267262.9735 \\
\hline 8 & 2057 & 18138456 & 97860996 & 15695080 & 1789168026 & 0.800723 & 557506.2217 \\
\hline 9 & 2869 & 39458250 & 235658250 & 34596378 & 4854767520 & 0.720962 & 1072635.935 \\
\hline 10 & 3871 & 79426050 & 520188900 & 70381650 & 11925036420 & 0.655616 & 1934279.19 \\
\hline 11 & 5083 & 150036084 & 1069197360 & 134113782 & 26999547234 & 0.601111 & 3307738.266 \\
\hline 12 & 6525 & 268799700 & 2070597360 & 242029500 & 57113643600 & 0.554959 & 5411348.628 \\
\hline 18 & 21127 & 4173529626 & 46591264656 & 3860392410 & 1938677864406 & 0.379858 & 55050387.67 \\
\hline 20 & 28741 & 8559729900 & 105428690700 & 7960596900 & 4880845917990 & 0.343696 & 101187112.4 \\
\hline
\end{tabular}

Table 2. to be continued 
Table 2. continued

\begin{tabular}{|r|r|r|r|r|r|r|r|}
\hline DEC-L & $N$ & $W$ & $W W$ & $r W$ & $S z$ & $J$ & $K f$ \\
\hline 2 & 7 & 26 & 31 & 16 & 51 & 3.250971 & 8.6315789 \\
\hline 3 & 23 & 494 & 810 & 518 & 2334 & 2.589834 & 96.2111649 \\
\hline 4 & 54 & 3810 & 7865 & 4776 & 32180 & 2.100464 & 512.1862408 \\
\hline 5 & 105 & 18390 & 45620 & 25290 & 242445 & 1.759945 & 1863.563395 \\
\hline 6 & 181 & 66310 & 191845 & 96590 & 1256855 & 1.51265 & 5352.32426 \\
\hline 7 & 287 & 195796 & 646796 & 296696 & 5044661 & 1.307104 & 13071.0489 \\
\hline 8 & 428 & 499764 & 1855290 & 779528 & 16801244 & 1.179465 & 28356.2922 \\
\hline 9 & 609 & 1141620 & 4703880 & 1820556 & 48537185 & 1.062183 & 56199.4209 \\
\hline 10 & 835 & 2389530 & 10819215 & 3877980 & 125351765 & 0.966031 & 103714.7037 \\
\hline 11 & 1111 & 4660370 & 23497590 & 7671730 & 295685885 & 0.885792 & 180664.5025 \\
\hline 12 & 1442 & 8574566 & 45799611 & 14282576 & 647213996 & 0.817829 & 300041.4421 \\
\hline 13 & 1833 & 15023034 & 86347820 & 25273638 & 1330444804 & 0.759532 & 478707.4656 \\
\hline 14 & 2289 & 25247430 & 155369045 & 42836586 & 2592556265 & 0.708979 & 738089.697 \\
\hline 15 & 2815 & 40934920 & 268527160 & 699964820 & 4824491710 & 0.664726 & 1104933.045 \\
\hline 16 & 3416 & 64328680 & 448095910 & 110655920 & 8624890840 & 0.625666 & 1612109.494 \\
\hline 17 & 4097 & 98355336 & 725024976 & 170145656 & 14885021806 & 0.590937 & 2299484.031 \\
\hline 18 & 4863 & 146770554 & 1141457775 & 255175848 & 24899518639 & 0.559856 & 3214837.188 \\
\hline 19 & 5719 & 214323990 & 1753760610 & 374298366 & 40508411920 & 0.531879 & 4414844.121 \\
\hline 20 & 6670 & 306944810 & 2636130205 & 538217560 & 64276669780 & 0.506563 & 5966110.248 \\
\hline
\end{tabular}

Table 2: Output of the MATLAB file showing the Wiener $(W)$, hyperWiener $(W W)$, reverseWiener $(r W)$, Szeged $(S z)$, Balaban $(J)$, and Kirchhoff $(K f)$ indices. The number of atoms $(N)$ and shells $(L)$ is also tabulated. 
MATLAB_R2014a. For the largest structures of 28741 atoms, the time was about 13 hours on the same computer. These results will vary with computer configuration, so they are meant to be estimates only. Table 2 lists the output of the program, after running all the coordinate files for the three types of clusters. The data for $L=1$ of the cuboctahedral cluster has very nearly integer values, with only the Kirchhoff index as a fraction at 6.5. Results of the program for carbon $C_{60}$ are shown in Table 2. The output agrees with previously reported data as given by references [17] and [26]. In [17], the authors report the Wiener index of $C_{60}$ as 8340, the Szeged index as 51840, the Balaban index as 0.91052, and the Kirchhoff index as 2294.2. Also, in [26] the author reports the hyper-Wiener index of $C_{60}$ as 27180 and the reverse Wiener index as 7590. As a result, we have some confidence our program is calculating the indices correctly.

Additionally, some results on the platonic solids have been published [27]. The only platonic solid we consider is the icosahedron and our results agree with the previous publication [27], if we remove the atom at the center, since our clusters have center-based atoms in the cluster. We might also mention, that the platonic solids have been made as clusters in nanogeometries, with most of the platonic solids formed in nano-gold shapes [5].

There have been extensive efforts at modeling fullerene data with the various indices [28-30]. Using the model of polynomial behavior for the Wiener index, we have obtained a seventh order polynomial fit

$$
T I(L)=a_{1} L+a_{2} L^{2}+a_{3} L^{3}+\cdots+a_{k} h L^{k}
$$

for the Wiener index of icosahedra, and the odd cuboctahedra function of $L$, as shown in Figure 3.

The least squares polynomial fit for these curves is shown in Table 3. The Wiener and reverse Wiener indices are modeled with seventh order polynomials, the Szeged with a ninth order polynomial, and the Kirchhoff with a sixth order polynomial. As noted in Table 1, the cuboctahedra need odd and even modeling. The even values of the Wiener index for cuboctahedra are hard to model. No polynomial up to degree nine yielded a reasonable fit. Also, while the icosahedral fit for the hyper-Wiener index can be modeled with an eighth order polynomial, the others could not. We have therefore left the hyper- Wiener modeling for future work. For the other modeling, the Chi square statistics are not zero even though the $R^{2}$ data equals one, but the Wiener index numbers range over nine orders of magnitude. If this were a perfect fit, the statistics would be better, so further analysis is needed. In our modeling, we choose a polynomial of lowest degree yielding a high quality fit. This analysis produces values of $\mathrm{k}$, the degree of the polynomial, for the different indices as

$$
\begin{array}{lll}
k=2 d_{T}+1, & d_{T} \geq 1, & \text { Wiener and reverse Wiener indices } \\
k=3 d_{T}, & d_{T} \geq 1, & \text { Szeged index } \\
k=2 d_{T}, & d_{T} \geq 1, & \text { Kirchoff index }
\end{array}
$$

where $d_{T}$ is the spatial dimension, (3) in our case. A conjecture on the asymptotic behavior of the Wiener index has been previously published [31]. The authors postulate that $W(L) \approx L^{k}$ where this is a polynomial with degree $k$ given by 


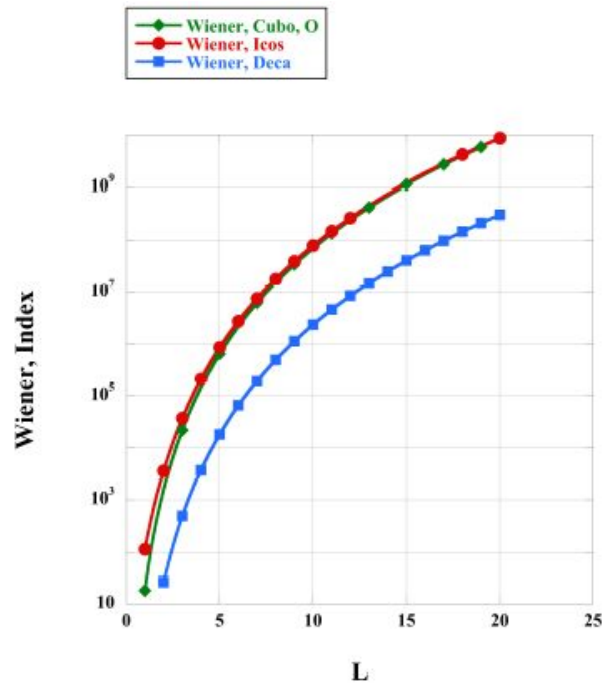

A. Seventh order polynomial fit for the Wiener index

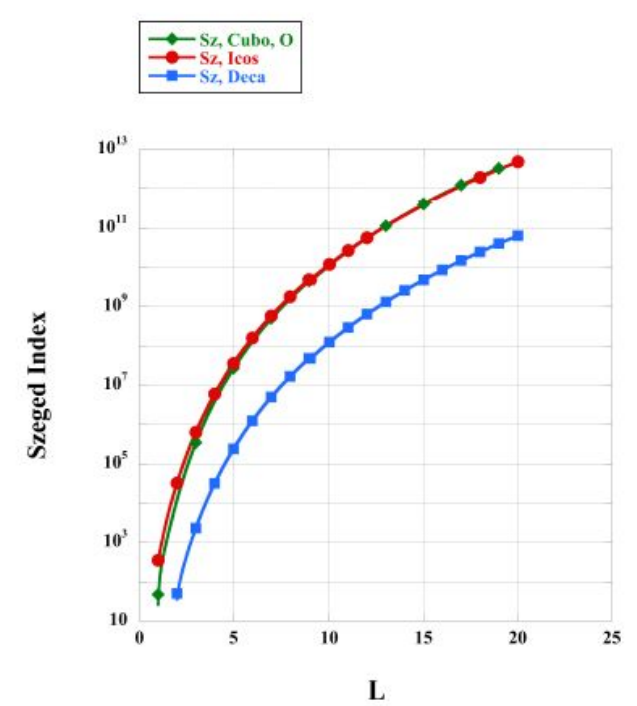

C. Ninth order polynomial fit for the Szeged index

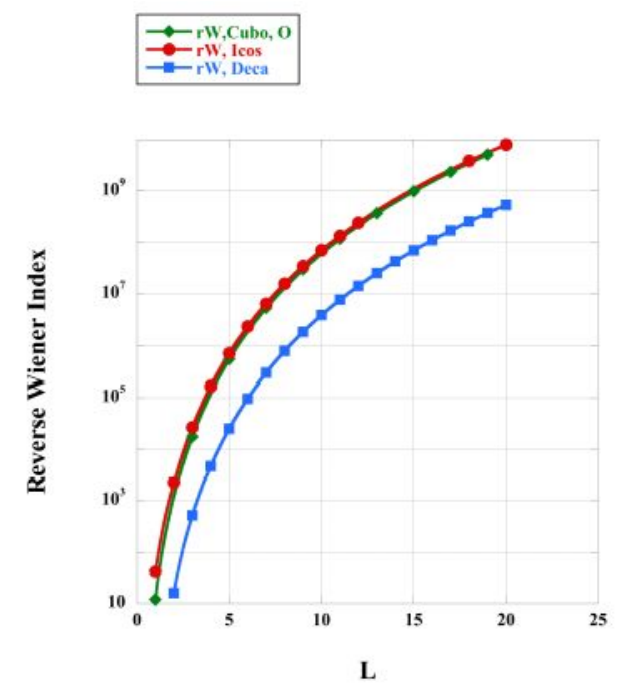

B. Seventh order polynomial fit for the reverse Wiener index

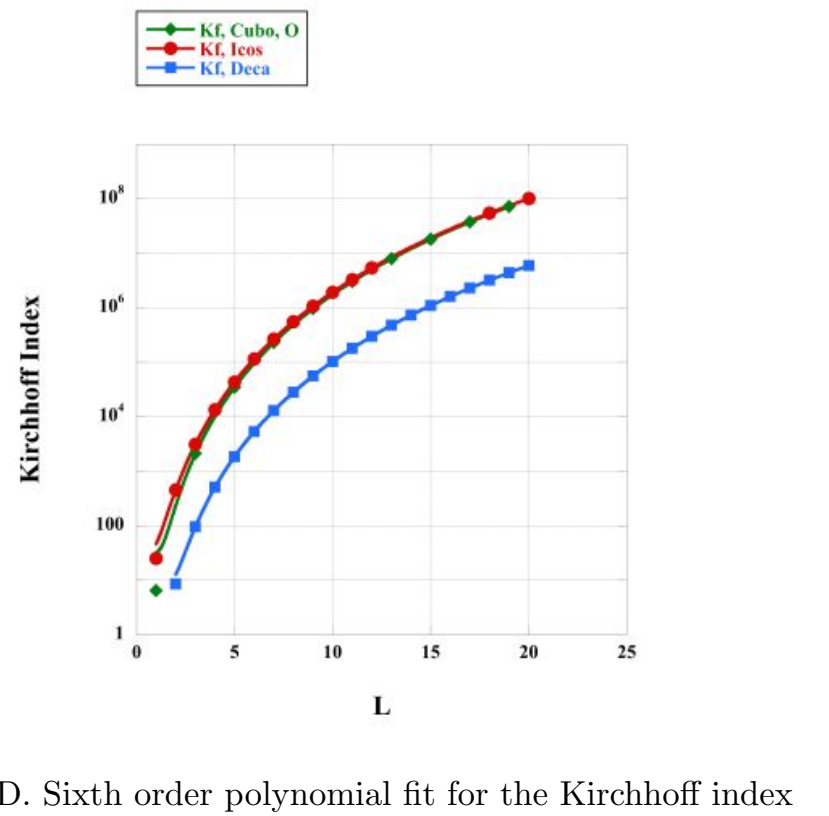

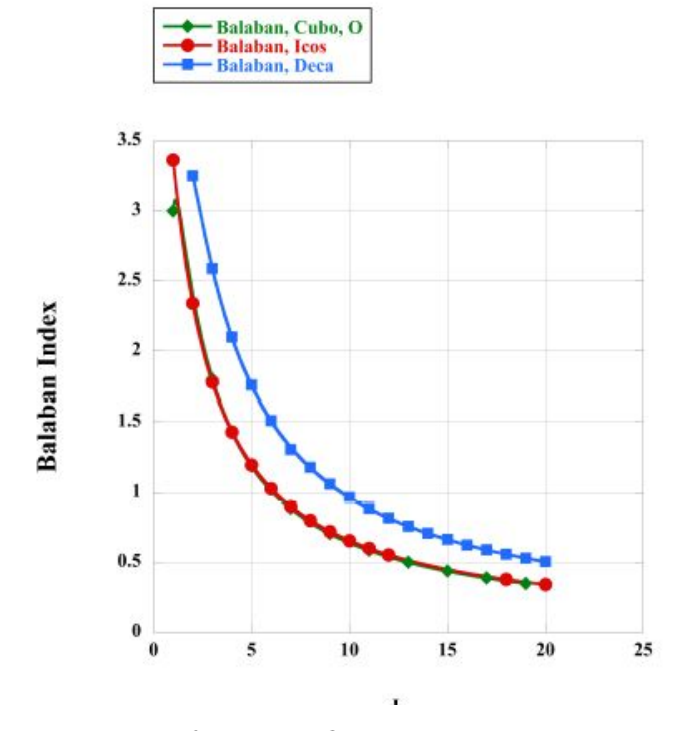

E. Inverse function fit to the Balaban index

Figure 3: Fitting the different indices of the clusters versus the number of shells $(L)$. 


\begin{tabular}{|c|c|c|c|c|c|c|}
\hline \multicolumn{7}{|c|}{ Wiener Index } \\
\hline & \multicolumn{2}{|c|}{ Icosahedron } & \multicolumn{2}{|c|}{ Decahedron } & \multicolumn{2}{|c|}{ Cubotahedron, Odd } \\
\hline & Value & Error & value & Error & Value & Error \\
\hline $\mathrm{a}$ & 6.5869 & 5.2537 & 0.40099 & 2.7643 & 7.9153 & 7.0388 \\
\hline $\mathrm{b}$ & -1.0373 & 4.7078 & -0.21208 & 2.0109 & -10.941 & 6.6485 \\
\hline $\mathrm{c}$ & 20.911 & 1.561 & -0.12672 & 0.55095 & 4.3409 & 2.3531 \\
\hline $\mathrm{d}$ & 31.219 & 0.24758 & 0.0026234 & 0.07396 & -4.5924 & 0.4011 \\
\hline $\mathrm{f}$ & 32.371 & 0.020085 & -0.11184 & 0.0052084 & 4.2131 & 0.035326 \\
\hline g & 19.665 & 0.00079937 & $4.93 \mathrm{E}-05$ & 0.00018461 & 12.094 & 0.0015472 \\
\hline $\mathrm{h}$ & 5.6191 & $1.23 \mathrm{E}-05$ & 0.24008 & $2.60 \mathrm{E}-06$ & 5.8287 & $2.66 \mathrm{E}-05$ \\
\hline$\chi^{2}$ & 43.196 & NA & 87.399 & NA & 15.354 & $\mathrm{NA}$ \\
\hline$R^{2}$ & 1 & NA & 1 & NA & 1 & $\mathrm{NA}$ \\
\hline \multicolumn{7}{|c|}{ Reverse Wiener Index } \\
\hline & \multicolumn{2}{|c|}{ Icosahedron } & \multicolumn{2}{|c|}{ Decahedron } & \multicolumn{2}{|c|}{ Cubotahedron, Odd } \\
\hline & Value & Error & value & Error & Value & Error \\
\hline $\mathrm{a}$ & 0.91866 & 1.6386 & -3.0755 & 9.0156 & -24.793 & 33.692 \\
\hline $\mathrm{b}$ & -4.0273 & 1.4683 & 2.7477 & 6.5584 & 29.12 & 27.913 \\
\hline $\mathrm{c}$ & 0.95659 & 0.48687 & 0.032369 & 1.7969 & -13.327 & 8.6911 \\
\hline $\mathrm{d}$ & 8.1745 & 0.077219 & -0.95409 & 0.24121 & 0.178 & 1.3081 \\
\hline $\mathrm{f}$ & 17.126 & 0.0062644 & 0.37594 & 0.016986 & 2.4986 & 0.10201 \\
\hline $\mathrm{g}$ & 13.666 & 0.00024932 & -0.69392 & 0.00060207 & 7.9078 & 0.0039637 \\
\hline $\mathrm{h}$ & 5.4921 & $3.8472 \mathrm{e}-6$ & 0.45436 & $8.4692 \mathrm{e}-6$ & 5.2824 & $6.0637 \mathrm{e}-5$ \\
\hline$\chi^{2}$ & 4.2018 & NA & 179.91 & NA & 706.99 & NA \\
\hline$R^{2}$ & 1 & NA & 1 & $\mathrm{NA}$ & 1 & $\mathrm{NA}$ \\
\hline \multicolumn{7}{|c|}{ Szeged Index } \\
\hline & \multicolumn{2}{|c|}{ Icosahedron } & \multicolumn{2}{|c|}{ Decahedron } & \multicolumn{2}{|c|}{ Cubotahedron, Odd } \\
\hline & Value & Error & value & Error & Value & Error \\
\hline $\mathrm{a}$ & -63.549 & 1124.2 & -563.08 & 657.12 & -1923.9 & 2478.2 \\
\hline $\mathrm{b}$ & 33.879 & 1740.2 & 687.18 & 712.84 & 3596.7 & 3744.6 \\
\hline $\mathrm{c}$ & 40.905 & 1030.9 & -328.15 & 306.2 & -2292.8 & 2086.4 \\
\hline $\mathrm{d}$ & 0.21209 & 311.27 & 81.852 & 69.11 & 710.24 & 580.31 \\
\hline $\mathrm{f}$ & 103.93 & 53.337 & -12.23 & 9.0836 & -120.98 & 90.901 \\
\hline $\mathrm{g}$ & 106.56 & 5.3809 & 1.0186 & 0.71958 & 13.502 & 8.3889 \\
\hline $\mathrm{h}$ & 76.842 & 0.31508 & -0.071729 & 0.033838 & 13.091 & 0.45213 \\
\hline $\bar{j}$ & 34.256 & 0.0098596 & 0.001443 & 0.00086959 & 21.278 & 0.013155 \\
\hline $\mathrm{k}$ & 7.614 & 0.00012702 & 0.12558 & $9.403 \mathrm{e}-6$ & 9.1091 & 0.0001595 \\
\hline$\chi^{2}$ & $2.0805 \mathrm{e}+5$ & NA & 71846 & $\mathrm{NA}$ & $2.7451 \mathrm{e}+5$ & $\mathrm{NA}$ \\
\hline$R^{2}$ & 1 & NA & 1 & NA & 1 & $\mathrm{NA}$ \\
\hline
\end{tabular}

Table 3 to be continued 
Table 3 continued

\begin{tabular}{|c|c|c|c|c|c|c|}
\hline \multicolumn{7}{|c|}{ Kirchoff Index } \\
\hline & \multicolumn{2}{|c|}{ Icosahedron } & \multicolumn{2}{|c|}{ Decahedron } & \multicolumn{2}{|c|}{ Cubotahedron, Odd } \\
\hline $\mathrm{a}$ & 80.585 & 17.708 & 23.637 & 3.3329 & 101.16 & 40.602 \\
\hline $\mathrm{b}$ & -84.813 & 11.216 & -20.718 & 1.8567 & -110.12 & 24.598 \\
\hline $\mathrm{c}$ & 42.212 & 2.5393 & 6.9709 & 0.37629 & 42.155 & 5.4263 \\
\hline d & 0.57419 & 0.25859 & -1.4567 & 0.035166 & -7.5149 & 0.54834 \\
\hline$f$ & 6.3829 & 0.012005 & 0.34066 & 0.001538 & 5.486 & 0.025759 \\
\hline $\mathrm{g}$ & 1.2557 & 0.00020666 & 0.07908 & $2.5479 \mathrm{e}-5$ & 1.2568 & 0.00045574 \\
\hline$\chi^{2}$ & 1546.5 & NA & 83.824 & NA & 2797.8 & $\mathrm{NA}$ \\
\hline$\overline{R^{2}}$ & 1 & NA & 1 & $\overline{\mathrm{NA}}$ & 1 & NA \\
\hline \multicolumn{7}{|c|}{ Balaban Index } \\
\hline & \multicolumn{2}{|c|}{ Icosahedron } & \multicolumn{2}{|c|}{ Decahedron } & \multicolumn{2}{|c|}{ Cubotahedron, Odd } \\
\hline $\mathrm{a}$ & 7.2052 & 0.0015778 & 10.675 & 0.037538 & 6.9586 & 0.0026281 \\
\hline $\mathrm{b}$ & -6.9239 & 0.0136 & -11.369 & 0.53103 & -5.807 & 0.031848 \\
\hline $\mathrm{c}$ & 4.4243 & 0.031297 & 11.901 & 2.0967 & 4.206 & 0.096456 \\
\hline $\mathrm{d}$ & -1.3414 & 0.019344 & -11.713 & 2.3864 & -2.3575 & 0.067379 \\
\hline$\chi^{2}$ & 2.92E-07 & NA & $5.76 \mathrm{E}-04$ & NA & $1.6294 \mathrm{e}-7$ & NA \\
\hline$R^{2}$ & 1 & NA & 0.99997 & $\mathrm{NA}$ & 1 & NA \\
\hline
\end{tabular}

Table 3: Fitting parameters for the polynomial topological indices and inverse models.

$$
k=2 d_{T}+1, \quad d_{T} \geq 1 .
$$

where $d_{T}$ is the spatial dimension of the graph. For $d_{T}=3$, this gives a polynomial of degree 7 , in agreement with what we find for the curve fits to the data. However, the coefficients of the polynomial remain best determined by the curve fitting we have done. These coefficients are given in Table 3.

The Balaban index is an exception to this type of analysis, as can be seen by the data in Table 2, where the index is shown to decrease with increasing $L$ and $N$. This data has been modeled using inverse functions for the icosahedral clusters, as shown in Figure 4. The curve in Figure 3.E is fit to the following function:

$$
J(L)=\frac{a}{L}+\frac{b}{L^{2}}+\frac{c}{L^{3}}+\frac{d}{L^{4}}
$$

where $a, b, c$, and $d$ are fit parameters and $L$ is the number of shells. If correct, the asymptote appears to be zero. The fit is quite good as shown by the $R^{2}$ statistics, which equals 1 and the Chi square is close to zero.

We have also looked at all the indices, as a function of the number of atoms, $N$. These plots fit extremely well to power law curves over twelve decades of output, and four decades of the number of atoms. These data are plotted in Figure 4. The exponents range from -0.29 


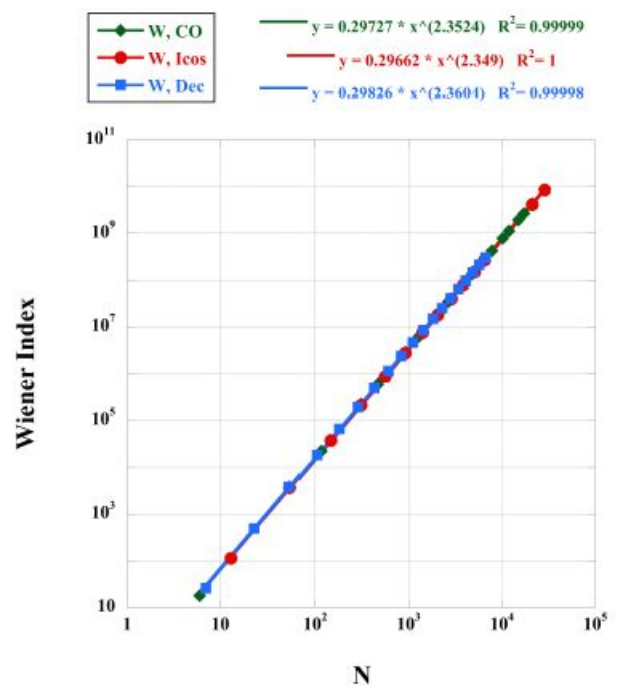

A. Wiener index

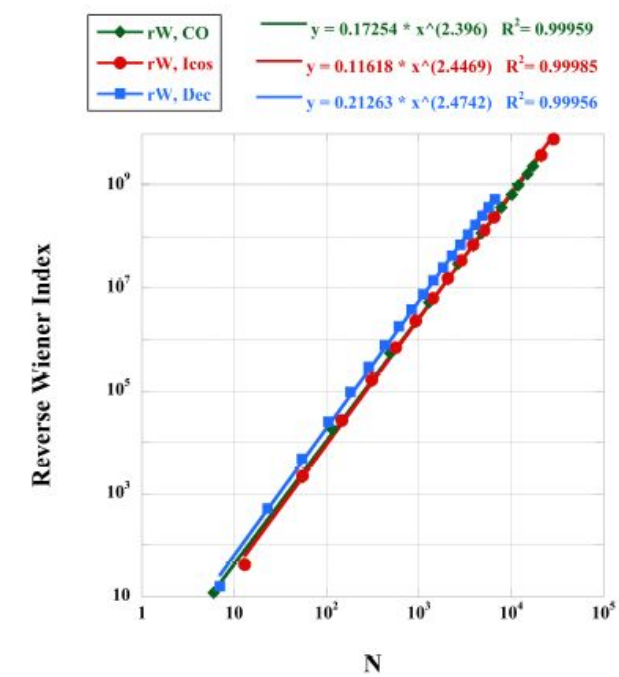

C. reverse Wiener index

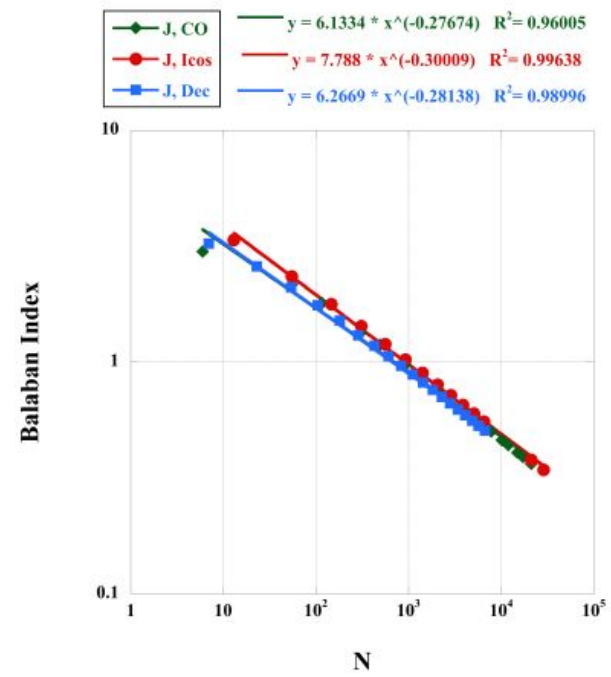

E. Balaban index

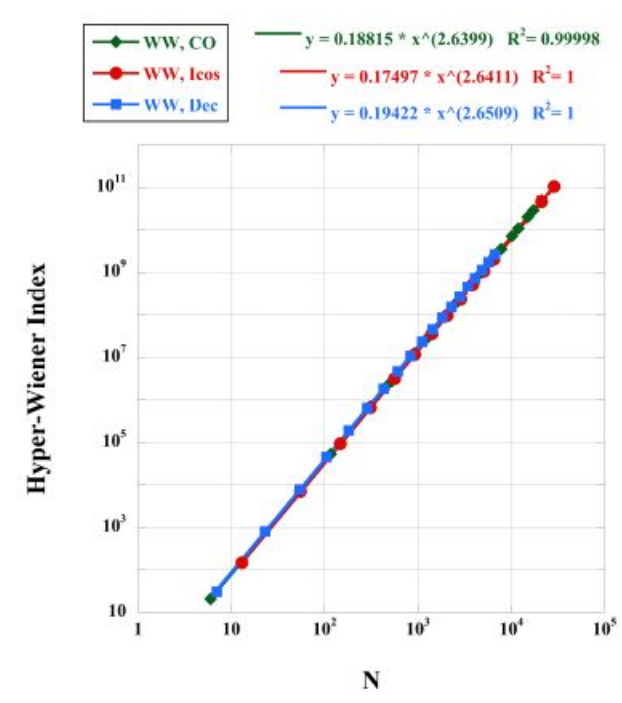

B. hyper Wiener index

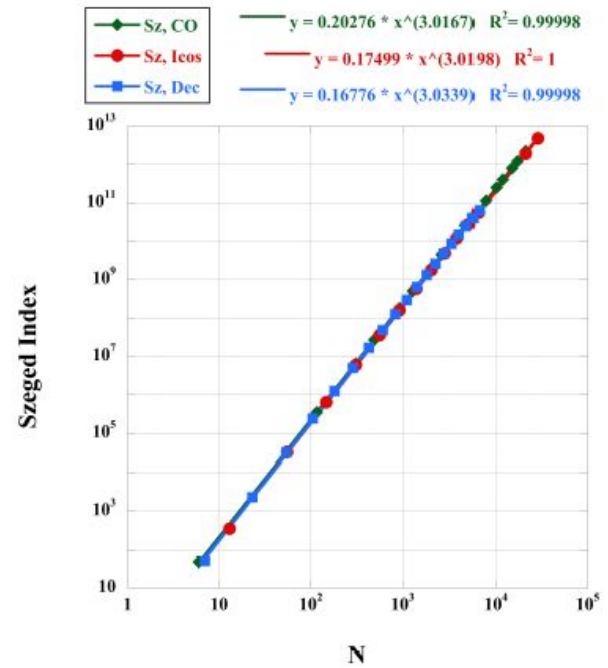

D. Szeged index

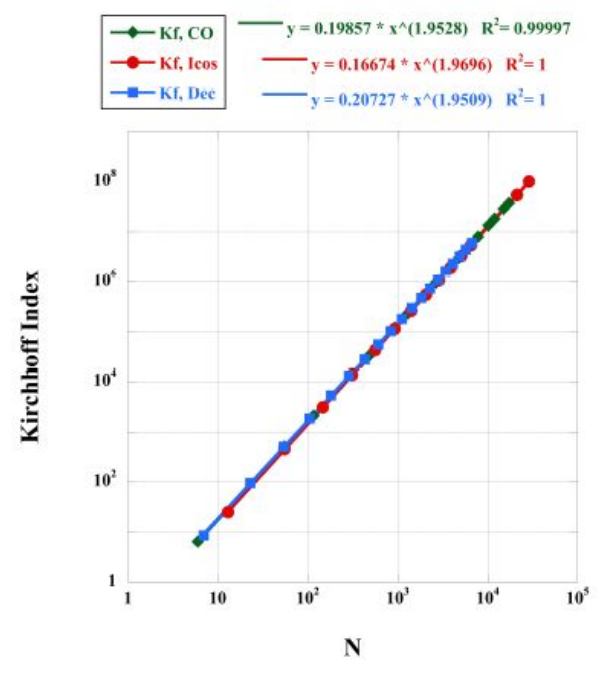

F. Kirchoff index

Figure 4: Power law of the indices as a function of the number of atoms $(N)$. 

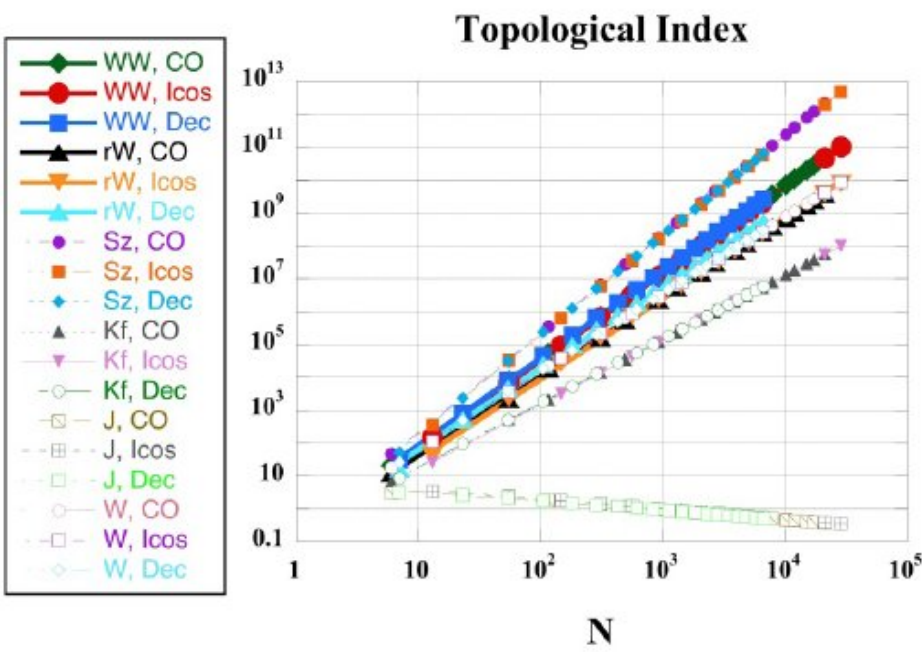

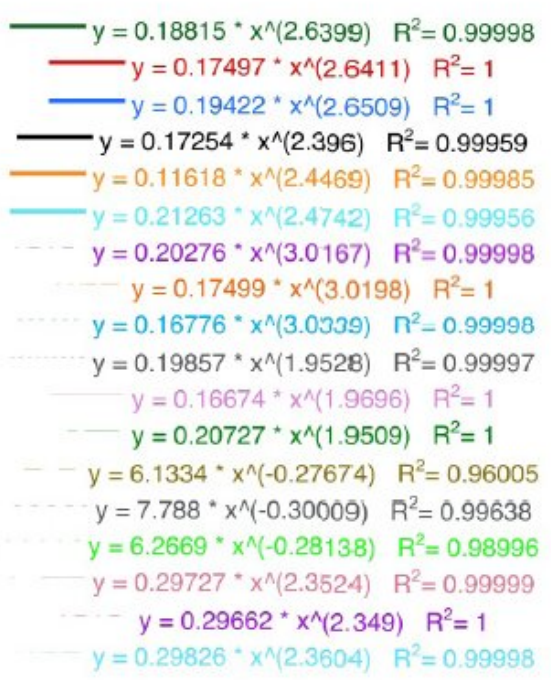

Figure 5: Power law plots of various indices as a function of $N$, the number of atoms.

to 3.01 and the coefficients vary from 0.11 to 7.788. A closer look at the data reveals that the exponent is associated with an index. The Wiener exponent in Fig. 4A is $2.35 \pm 0.01$, the hyper-Wiener index in Fig. $4 \mathrm{~B}$ has an exponent of $2.64 \pm 0.01$, the reverse Wiener exponent in Fig. $4 \mathrm{C}$ is $2.43 \pm 0.04$, the Szeged index in Fig. 4D has the largest exponent at $3.02 \pm 0.01$, the Balaban exponent in Fig. $4 \mathrm{E}$ is $-0.29 \pm 0.02$, and the Kirchhoff exponent in Fig. $4 \mathrm{~F}$ is $1.95 \pm 0.01$. Evidently, it does not matter what type of cluster it is, as all the exponents and the coefficients are nearly identical within each index, with the reverse Wiener index showing the most variation. We have previously published Wiener index data for fullerenes showing that they have similar behavior for power law analysis [32].

The asymptotic limit in $N$ for the topological indices can be approximated. In [31], the authors conjecture that for the Wiener index, we have

$$
\lim _{N \rightarrow \infty} W(N)=a N^{s}
$$

where $s$ is

$$
s=2+1 / d_{T} .
$$

As originally written, equations (16) and (17) apply to the Wiener index, and we generalize to any topological index, although equation (17) will be different for each index. In a separate work [33], we have determined equations for $s$ as $d_{T}$ changes, for the six topological indices, although the geometry of the clusters is different. In equation (16), both $a$ and $s$ can be numerically interpolated [31] from data in Figure 4 and Table 2, using adjacent ordered pairs of $(T I, N)$, where $T I$ is a topological index as $N \rightarrow \infty$. In other words, we determine the values of $a$ and $s$ by looking at the limit of these values in the power law, as $N$ gets large. We find the following asymptotic behavior for the topological indices 


$$
\begin{array}{rlrl}
W(N) & \approx \frac{1}{3} N^{7 / 3}, & & r W(N) \approx \frac{1}{4} N^{7 / 3} \\
S z(N) & \approx \frac{1}{5} N^{3}, & W W(N) \approx \frac{3}{20} N^{8 / 3} \\
K f(N) \approx \frac{3}{20} N^{2}, & J(N) \approx \frac{19}{2} N^{-1 / 3} .
\end{array}
$$

Equations (18) represent the best fits we have to the data, but there remains the possibility that corrections could be made with more accurate data. The asymptotic exponent for the Wiener index is $s=7 / 3$, in agreement with the conjecture in [31] and equation (17) for $d_{T}=3$. Our numerical calculations have thus experimentally verified some of the results of reference [31]. Given this data, it is not surprising that the indices have polynomial behavior with $L$, since $N$ is a cubic function in $L$, as shown in Table 1 . In fact, the degree of the polynomial is exactly as predicted after examining the power law dependence and the cubic behavior in $N$ with $L$. This reflects the fact that $T I(L)$ is a composition of $T I(N)$ and $N(L)$.

\section{Conclusion}

We have presented the first data to enumerate several topological indices for three types of metal nanoclusters, the icosahedral, cuboctahedral, and decahedral symmetries. The cuboctahedral clusters require even and odd modeling as a function of the number of shells, while the icosahedral and decahedral clusters do not. It is shown that many of the indices can be modeled with higher degree polynomials, while the Balaban index requires inverse functions. All indices can also be modeled with power law curves, as a function of $N$, the number of atoms. The magnitude of the exponent is associated with the type of index. The asymptotic limits of the topological indices are determined as a function of $N$. A conjecture of the asymptotic behavior for the Wiener index is experimentally confirmed.

Acknowledgements We thank the anonymous reviewers for pointing out reference [31] and the editors for comments that improved the manuscript.

\section{References}

[1] B.K. Teo and N.J.A. Sloane, Magic numbers in polygonal and polyhedral clusters, Inorg. Chem. 24, 4545-4558 (1985).

[2] J.M. Montejano-Carrizales, F. Aguilera-Granja, and J.L. Moran-Lopez, Direct enumeration of the geometrical characteristics of clusters, NanoStructured Mater. 8(3), 269-287 (1997).

[3] J. Chen, B. Lim, E.P. Lee, and Y. Xia, Shape-controlled synthesis of platinum nanocrystals for catalytic and electrocatalytic applications, Nano Today 4, 81-95 (2009).

[4] W. Niu and G. Xu, Crystallographic control of noble metal nanocrystals, Nano Today 6 , 
265-285 (2011).

[5] F. Kim, S. Connor, H. Song, T. Kuykendall and P. Yang, Platonic gold nanocrystals, Angew. Chem. 116, 3673-3677 (2004).

[6] K. M. Bratlie, H. Lee, K. Komvopoulos, P. Yang, and G. A. Somorjai, Platinum nanoparticle shape effects on benzene hydrogenation selectivity, Nano Lett. 7(10), 3097-3101 (2007).

[7] A. Sanchez-Iglesias, I. Pastoriza-Santos, J. Perez-Juste, B. Rodriguez-Gonzalez, F. Javier Garcia de Abajo, and L.M. Liz-Marzan, Synthesis and optical properties of gold nanodecahedra with size control, Adv. Mater. 18, 2529-2534 (2006).

[8] H. Wiener, Structural determination of paraffin boiling points, J. Am. Chem. Soc. 69, 17-20 (1947).

[9] H. Hosoya, A newly proposed quantity characterizing the topological nature of structural isomers of saturated hydrocarbons, Bull. Chem. Soc. Japan, 44, 2332-2339 (1971).

[10] P. Schwerdtfeger, L. Wirz, and J. Avery, Program fullerene: a software package for constructing and analyzing structures of regular fullerenes, J. Comput. Chem. 34, 1508-1526 (2013).

[11] F. Kaatz and A. Bultheel, Informational thermodynamic model for nanostructures, $J$. Comput. Chem. 52(6), 1563-1575 (2014).

[12] F. Kaatz and A. Bultheel, Statistical mechanics of two dimensional tilings, Physica A. 391(6), 2957-1963 (2012).

[13] A. Mohajeri, M Alipour, and M. B. Ahmadi, A graph theory study on $(\mathrm{ZnS})_{n}(n=3-10)$ nanoclusters Chem. Phys. Lett. 503, 162-166 (2011).

[14] J.M. Amigo, J. Galvez, V.M. Villar, review on molecular topology: applying graph theory to drug discovery and design, Naturwissenschaften 96, 749-761 (2009).

[15] W.R. Müller, K. Szymanski, J.V. Knop, and N. Trinajstic, An algorithm for construction of the molecular distance matrix, J. Comput. Chem. 8(2), 170-173 (1987).

[16] B. Zhou and N. Trinajstic, Maximum eigenvalues of the reciprocal distance matrix and the reverse Wiener matrix, Inter. J. Quan. Chem. 108, 858-864 (2008).

[17] I. Gutman, Graph Theory Notes New York 27, 9-15 (1994).

[18] P.W. Fowler, G. Caporossi, and P. Hansen, Distance matrices, Wiener indices, and related invariants of fullerenes, J. Phys. Chem. A 105, 6232-6242 (2001).

[19] P.V. Khadikar, N.V. Deshpande, P.P. Kale, A. Dobrynin, I. Gutman, and G. Domotor, The Szeged index and an analogy with the Wiener index, J. Chem. Inf. Comput. Sci. 35, $547-550$ (1995).

[20] S. Klavzar and M.J. Nadjafi-Arani, Wiener index versus Szeged index in networks, Discr. Appl. Math. 161, 1150-1153 (2013).

[21] A.T. Balaban, Highly discriminating distance-based topological index, Chem. Phys. Lett. 89(5), 399-404 (1982).

[22] A.T. Balaban, Topological indices based on topological distances in molecular graphs, Pure Appl. Chem. 55(2), 199-206 (1983). 
[23] A.T. Balaban, D. Mills, O. Ivanciuc, and S.C. Basak, Reverse Wiener indices, Croat. Chem. Acta 73(4), 923-941 (2000).

[24] E. Estrada, and N. Hatano, Topological atomic displacements, Kirchhoff and Wiener indices of molecules, Chem. Phys. Lett. 486, 166 (2010).

[25] I. Gutman and B. Mohar, The quasi-Wiener and the Kirchhoff indices coincide, J. Chem. Inf. Comput. Sci. 36, 982-985 (1996).

[26] M. Arezoomand, On the distance-based topological indices of fullerenes and fullerenyl anions having dendrimer units, Digest Journal of Nanomaterials and Biostructures 4(4), 713-722 (2009).

[27] D. Babić, D.J. Klein, I. Lukovits, S. Nikolić, and N. Trinajstić, Resistance-distance matrix: a computational algorithm and its application, Inter. J. Quan. Chem. 90, 166-176 (2002).

[28] M. Faghani and A.R. Ashrafi, Resistance-distance matrix: a computational algorithm and its application, Fullerenes, Nanotubes and Carbon Nanostructures 21(7), 561-567 (2013).

[29] A.R. Ashrafi, M. Ghorbani, and M. Jalali, The vertex PI and Szeged indices of an infinite family of fullerenes, J. Of Theoretical and Comput. Chem. 7(2), 221-231 (2008).

[30] Y. Alizadeh, A. Iranmanesh, and S. Klavzar, Interpolation method and topological indices: the case of fullerenes $\mathrm{C}_{12 k+4}$, MATCH Commun. Math. Comput. Chem. 68, 303-310 (2012).

[31] O. Ori, F. Cataldo, D Vukičević, A. Graovac, Wiener Way to Dimensionality, Iranian J. Math. Chem. 1(2), 5-15 (2010).

[32] F. Kaatz and A. Bultheel, Statistical properties of carbon nanostructures, J. Math. Chem. 51(5), 1211-1220 (2013).

[33] F. Kaatz and A. Bultheel, Dimensionality and graph properties of hypercube clusters, unpublished. 